\title{
OTIOTOMICS
}

Revista de economía, empresa y sociedad

\section{Wikipedia en la universidad: una guía de buenas prácticas}

\section{Antoni Meseguer-Artola}

Profesor de los Estudios de Economía y Empresa (UOC)

RESUMEN Aunque Wikipedia es una fuente inicial de información ampliamente utilizada por estudiantes de cualquier nivel académico, es difícil encontrar cursos de educación superior en los que Wikipedia tenga un papel activo en el proceso de aprendizaje. A partir de los principales resultados del proyecto Wiki4HE, en este artículo se hace una breve descripción de los factores más importantes que influyen en la decisión de los profesores a la hora de usar Wikipedia en las aulas en su acción docente. Además de los factores estrictamente tecnológicos, relacionados con la usabilidad y la utilidad de la plataforma de trabajo que proporciona la enciclopedia, se exponen los factores académicos y profesionales que tienen una mayor implicación: la percepción de la calidad de Wikipedia, su imagen social, el perfil 2.0 del profesorado y su actitud colaborativa, así como el reconocimiento institucional del uso de estas herramientas.

Este artículo tiene dos objetivos principales. El primer objetivo es destacar la importancia de la opinión que tienen los compañeros académicos sobre Wikipedia a la hora de que un profesor tome la decisión de usar esta enciclopedia de manera activa en la docencia. El segundo objetivo es mostrar el papel que puede tener en esta decisión el disponer de una guía de buenas prácticas, que recoja de forma sistemática las experiencias de otros profesores y que permita mejorar la imagen social de Wikipedia.

PALABRAS CLAVE Wikipedia; educación superior; docencia; buenas prácticas; competencias; calidad

\section{Wikipedia in universities: a guide of good practices}

\begin{abstract}
Although Wikipedia is an information source used extensively by students at all academic levels, it is hard to find higher education courses in which Wikipedia has a formal role in the learning process. Using the principal results of the Wiki4HE project as its foundation, this article briefly describes key factors that influence the decisions of academic staff regarding the use of Wikipedia in their teaching. In addition to technological factors relating to the user-friendliness and utility of the work platform this encyclopaedia provides, it also examines the academic and professional factors that have a greater influence: the perceived quality of Wikipedia, its social image, the 2.0 profile of academic staff and their collaborative attitudes, and institutional recognition of these resources.
\end{abstract}


The article has two main objectives: the first is to highlight the importance of the opinions academic colleagues have of Wikipedia, and the influence this has on decisions to use the encyclopaedia actively in teaching; the second is to show how this decision could be affected by access to a guide to good practice, one that systematically brought together the experiences of other academic staff and enabled an improvement in Wikipedia's social image.

KEYWORDS Wikipedia; higher education; teaching; good practice; competencies; quality

\section{Introducción}

La gran disponibilidad de contenidos educativos abiertos es uno de los mayores impactos que internet está teniendo en la educación universitaria. El movimiento para los Recursos Educativos Abiertos se puso en marcha en el MIT en 2001, y en pocos años esta iniciativa ha estimulado a muchas otras universidades a hacer los recursos didácticos (materiales docentes, guías de estudio, colecciones de ejercicios, etc.) accesibles para todo el mundo a través de la red.

La aparición de la llamada web 2.0 ha permitido potenciar el efecto de este movimiento. La utilización del amplio abanico de soluciones tecnológicas que se proponen ha facilitado el uso de estos contenidos en abierto en los procesos de aprendizaje, así como la propia generación de contenidos. Wikipedia se encuentra precisamente en el punto de intersección entre estos dos movimientos (Aibar et al., 2015). Por un lado, podemos considerar esta enciclopedia como un amplio repositorio virtual de información y conocimiento, pero también la podemos considerar como una plataforma tecnológica que, aprovechando las herramientas 2.0, facilita la colaboración para la construcción colectiva del conocimiento.

Aunque Wikipedia es uno de los recursos más utilizados a nivel individual por los estudiantes y los profesores en la enseñanza superior (Wannemacher y Schulenburg, 2010), no son muchos los cursos que abiertamente integran su uso en el proceso de aprendizaje. Aunque a menudo la actitud del profesorado universitario no es muy positiva (Dooley, 2010), cada vez hay un número creciente de profesores de diversas universidades de todo el mundo (como por ejemplo la UOC) que están utilizando Wikipedia como herramienta para la enseñanza.

Las principales preocupaciones que tienen los docentes sobre Wikipedia son la calidad, la precisión y la fiabilidad. En general, hay un gran escepticismo sobre Wikipedia como una fuente legítima de información (Dooley, 2010). Por ejemplo, la falta de una autoría clara e identificable, y la dificultad para verificar el contenido de los artículos, son una de las principales razones para no citar Wikipedia en artículos académicos (Jaschik, 2007).

En este artículo veremos que hay muchos factores que influyen en el uso docente de Wikipedia, pero nos centraremos muy especialmente en los relativos al entorno académico y profesional del profesor. Explicaremos cómo la opinión de los colegas de ámbito de conocimiento sobre Wikipedia, así como la existencia de ejemplos de buenas prácticas, son elementos muy importantes a la hora de tomar la decisión de usar activamente la enciclopedia en las aulas. Los resultados positivos en el rendimiento de los estudiantes que se están obteniendo en las experiencias recogidas en la guía (por ejemplo, Meseguer-Artola, 2014) también ayudan a ir cambiando la visión negativa que muchos profesores podían tener inicialmente.

\section{Factores que influyen en el uso docente de Wikipedia}

En el proyecto «The use of Internet open content for university education: an empirical study on the perceptions, attitudes and practices of university faculty on Wikipedia» (Wiki4HE, http://oer.uoc.edu/wiki4HE/about/), que se ha desarrollado en la Universitat Oberta de Catalunya (UOC), con la financiación del programa Recercaixa-2011, se ha analizado cuáles eran los principales factores que podían determinar el uso docente de Wikipedia. A partir 
de la opinión de 800 profesores y colaboradores docentes de la UOC, de ámbitos de conocimiento muy diversos y con una experiencia docente media de 10,4 años, se ha podido demostrar que aparte de aspectos técnicos relacionados con la usabilidad y la utilidad de Wikipedia, hay otros factores externos que desempeñan un papel muy importante (ver figura 1).

Figura 1. Factores que influyen en el uso docente de Wikipedia en la educación superior

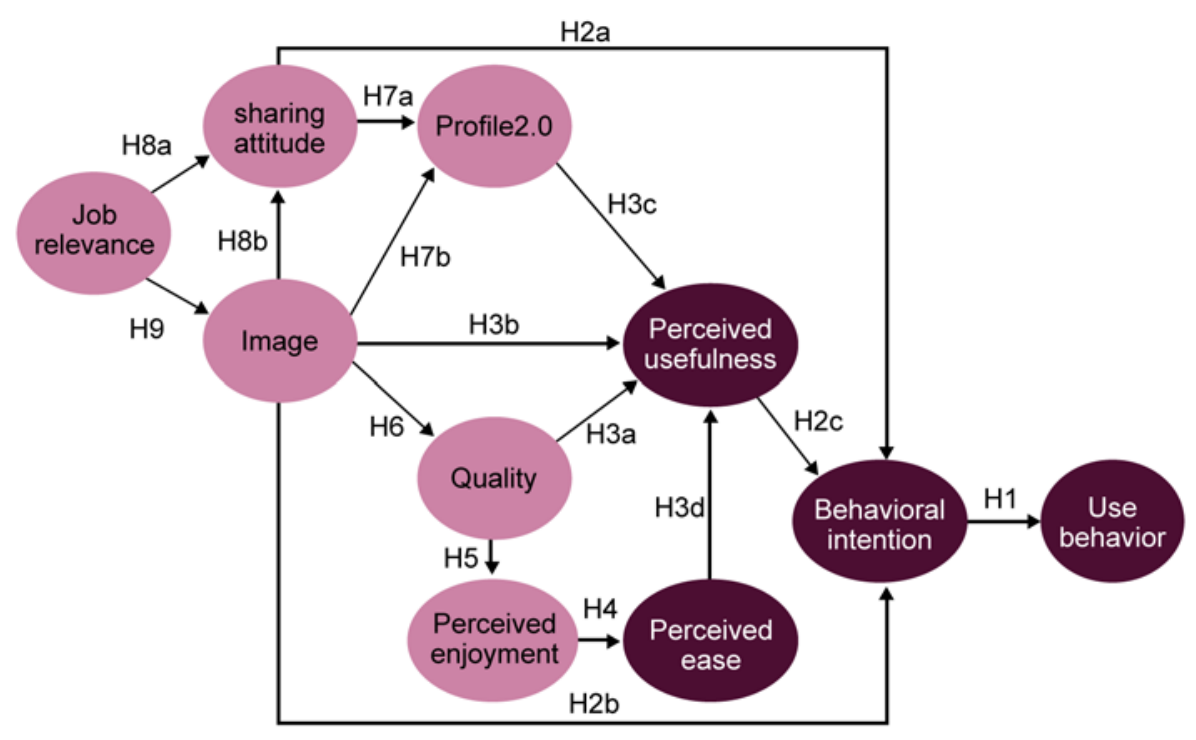

Fuente: Meseguer-Artola et al. (2015).

En este proyecto se pone de manifiesto que la percepción de la calidad influye positivamente en la utilidad percibida de Wikipedia, y por tanto tiene también un impacto sobre la decisión de usar Wikipedia como parte del proceso de enseñanza. Aunque la calidad se presenta en la literatura académica como uno de los principales factores que explican las actitudes y las prácticas del profesorado hacia Wikipedia (Chen, 2010), se han encontrado otros factores externos que son clave en la explicación del uso de Wikipedia con finalidad didáctica: la imagen social de la enciclopedia en el mundo académico, las actitudes del profesorado hacia la publicación de recursos abiertos y hacia el uso de entornos de colaboración, y el reconocimiento institucional y el apoyo que reciben los profesores por parte de su propia universidad cuando se emplean plataformas abiertas como Wikipedia (Bayliss, 2013).

Los resultados analizados indican que, además de la propia percepción sobre la calidad de la enciclopedia, la percepción de la opinión de los compañeros académicos tiene un papel fundamental a la hora de tomar la decisión de usar Wikipedia de forma activa en la acción docente (Llados et al., 2013). Si nuestros compañeros tienen una visión positiva de Wikipedia y hacen un uso docente de ella, tendremos más propensión a usarla también en nuestras aulas (Black, 2008; Eijkman, 2010). Como se puede ver en la figura 1, la utilidad está directamente condicionada por la propia percepción de la calidad y por la imagen social que uno cree que tiene. Por tanto, los compañeros actúan claramente como modelos de conducta para la mayoría de los miembros del profesorado (Meseguer-Artola et al., 2015).

Si analizamos en detalle las valoraciones de los profesores sobre la imagen social que tiene Wikipedia, podemos comprobar que aunque no está muy mal visto compartir recursos en abierto a través de plataformas en abierto como Wikipedia (solo un 24\% de los profesores está totalmente en desacuerdo o simplemente en desacuerdo), la opinión que creen los profesores que tienen sus compañeros sobre Wikipedia no es muy positiva (ver tabla 1). Solo un $14 \%$ de los profesores está de acuerdo o completamente de acuerdo en que Wikipedia está bien vista entre los compañeros. Asimismo, solo un 25\% de los profesores considera que sus compañeros utilizan Wikipedia. 
Tabla 1. Valoración sobre la imagen social de Wikipedia (\%)

\begin{tabular}{|c|c|c|c|c|c|}
\hline & $\begin{array}{l}\text { Totalmente en } \\
\text { desacuerdo }\end{array}$ & 2 & 3 & 4 & $\begin{array}{l}\text { Totalmente } \\
\text { de acuerdo }\end{array}$ \\
\hline $\begin{array}{l}\text { Wikipedia está bien vista } \\
\text { entre los compañeros }\end{array}$ & 14,83 & 37,72 & 33,76 & 11,00 & 2,69 \\
\hline $\begin{array}{l}\text { Compartir recursos docentes } \\
\text { en abierto está bien visto }\end{array}$ & 3,71 & 19,82 & 31,20 & 33,50 & 11,76 \\
\hline $\begin{array}{l}\text { Los compañeros utilizan } \\
\text { Wikipedia }\end{array}$ & 8,24 & 26,06 & 40,43 & 20,48 & 4,79 \\
\hline
\end{tabular}

Fuente: Proyecto Wiki4HE.

Aunque de los resultados de la encuesta se desprende que hay un porcentaje bastante alto de profesores que consideran Wikipedia como una herramienta muy interesante y con mucho potencial (solo un $20 \%$ considera que no es útil para la docencia), hay muy pocos profesores que la estén usando de manera activa en su acción docente. Solo un $10 \%$ la han utilizado en el momento de preparar materiales docentes o en las actividades que deben realizar los estudiantes. De estos resultados se desprende que hay un salto muy grande entre la opinión sobre las potencialidades de Wikipedia y su uso efectivo en la docencia. En el marco del proyecto se analizan también una serie de incentivos para tratar de acercar un poco estas posiciones tan distantes.

Entre los diferentes incentivos sobre los que se preguntó a los profesores, el disponer de un catálogo de buenas prácticas fue uno de los mejores valorados (ver tabla 2). Más de un $65 \%$ de los profesores estaba de acuerdo o muy de acuerdo en considerar que disponer de una guía de buenas prácticas es un claro incentivo para empezar a usar Wikipedia en la docencia. Aparte de este, también se valoraba muy positivamente poder tener un compañero que explicara su experiencia, poder recibir formación específica o que estas prácticas innovadoras tuvieran un mayor reconocimiento institucional.

Tabla 2. Valoración sobre los principales incentivos para el uso docente de Wikipedia (\%)

\begin{tabular}{|l|c|c|c|c|c|}
\hline & $\begin{array}{c}\text { Totalmente } \\
\text { en } \\
\text { desacuerdo }\end{array}$ & $\mathbf{2}$ & $\mathbf{3}$ & $\begin{array}{c}\text { Totalmente } \\
\text { de acuerdo }\end{array}$ \\
\hline Catálogo de buenas prácticas & 4,40 & 6,48 & 23,19 & 35,75 & 30,18 \\
\hline $\begin{array}{l}\text { Compañero explica la } \\
\text { experiencia }\end{array}$ & 5,31 & 12,18 & 29,92 & 31,09 & 21,50 \\
\hline Recibir formación específica & 5,45 & 13,38 & 30,26 & 28,44 & 22,47 \\
\hline Reconocimiento institucional & 6,54 & 10,98 & 29,54 & 28,10 & 24,84 \\
\hline
\end{tabular}

Fuente: Proyecto Wiki4HE.

Teniendo en cuenta estos resultados, y con el objetivo de alentar el uso docente de Wikipedia en la educación superior, se consideró muy conveniente elaborar una recopilación de buenas prácticas de profesores que ya han tenido una experiencia previa y hacer difusión de ellas. En el siguiente apartado se presenta esta guía, que se elaboró en el marco del proyecto Wiki4HE. 


\section{Guía de buenas prácticas docentes}

La guía de buenas prácticas del proyecto de investigación Wiki4HE permite dar a conocer un abanico muy amplio de experiencias docentes que se han llevado a cabo en todo el mundo con Wikipedia, y mostrar los efectos que ha tenido en el rendimiento académico de los estudiantes su uso activo. Esta guía pretende documentar las buenas prácticas que se han hecho en trabajos académicos, informes o presentaciones, y complementa la información que se puede encontrar en la propia Wikipedia sobre más de 500 proyectos educativos que se han desarrollado en muchas universidades del mundo (como por ejemplo Harvard, Yale o el MIT)'. Tal y como se explica en la guía, en estos proyectos han participado más de 4.500 estudiantes y se estima que han añadido a Wikipedia (y en varios idiomas) el equivalente a 20.000 páginas impresas de contenido, en más de 6.000 artículos.

Esta guía muestra que la utilización de Wikipedia como herramienta docente implica en la gran mayoría de los casos la realización de actividades individuales o grupales por parte de los estudiantes, en las que se crean artículos nuevos o se mejoran y/o corrigen artículos existentes. Los beneficios educativos de estas actividades de aprendizaje implican mejoras en el rendimiento académico de los estudiantes y se concretan en la mejora y profundización de muchas competencias asociadas a la mayor parte de estudios universitarios: trabajo colaborativo y en equipo, escritura y comprensión, análisis y síntesis, etc. La guía de buenas prácticas se puede encontrar a través del siguiente enlace:

\section{http://hdl.handle.net/10609/41661}

El objetivo principal de la recopilación de prácticas es ofrecer de manera sistemática una serie de recomendaciones para docentes universitarios que o bien quieran profundizar en la utilización docente de Wikipedia, o bien se quieran iniciar en su uso. Estas recomendaciones se han recogido a partir de la experiencia de diferentes profesores, en diversos ámbitos de conocimiento y en varios niveles universitarios. Cada experiencia tiene una ficha en la que se da una breve descripción del curso, información sobre el proceso de evaluación que se ha desarrollado y una valoración final de la experiencia.

Teniendo en cuenta que la valoración general es mayoritariamente positiva, esta guía puede ser una herramienta muy útil tanto a la hora de diseñar nuevos cursos como a la hora de proponer innovaciones docentes en cursos que ya están en funcionamiento. En la parte final de la guía se destacan cuáles son los principales factores de éxito que han hecho que la docencia se llevara a cabo de manera satisfactoria (por ejemplo, incluir información clara y detallada sobre las normas y el proceso de edición en Wikipedia). También se detallan cuáles han sido las principales dificultades encontradas por los profesores a la hora de usar Wikipedia en sus aulas.

\section{Conclusiones}

A partir de los principales resultados del proyecto Wiki4HE, en este artículo se han descrito los factores más importantes que influyen en la decisión de los profesores a la hora de usar Wikipedia en las aulas en su acción docente. Además de los factores estrictamente tecnológicos, relacionados con la usabilidad y la utilidad de la plataforma de trabajo que proporciona la enciclopedia, se muestra que hay otros factores, académicos y profesionales, que tienen también una importante implicación.

La percepción de la calidad influye positivamente en la utilidad percibida de Wikipedia, y por tanto tiene también un impacto sobre la decisión de usar esta enciclopedia. Este es uno de los factores más importantes a la hora de explicar las actitudes y las prácticas del profesorado hacia Wikipedia. Pero no es el único. Entre los

1. http://en.wikipedia.org/wiki/Wikipedia:Education_program 
diferentes factores que se comentan en este artículo (como por ejemplo el perfil 2.0 del profesorado y su actitud colaborativa, o el reconocimiento institucional del uso de estas herramientas), destaca la imagen social que se tiene de la enciclopedia. La opinión que tienen los compañeros académicos sobre Wikipedia impacta de forma directa en el momento en el que un profesor toma la decisión de usar o no esta enciclopedia de manera activa en la acción docente.

Hemos visto cómo un porcentaje muy bajo de profesores cree que Wikipedia está bien vista entre sus compañeros y que además la están utilizando en su acción docente. Aunque lo ven como una herramienta muy interesante y con mucho potencial (Aibar et al., 2015), son muy pocos los que finalmente la están utilizando. Para aproximar estas posiciones tan separadas, se ha visto que disponer de una guía de buenas prácticas docentes puede ser una herramienta muy importante. Con la recopilación, de forma sistemática, de experiencias positivas que han tenido otros profesores se puede mejorar la imagen social de Wikipedia. Y, en consecuencia, se puede incrementar su uso docente en la educación superior.

En la última parte del artículo se explica cómo es la guía de buenas prácticas que se ha elaborado en el marco del proyecto Wiki4HE. Esta guía muestra diferentes maneras de utilizar Wikipedia en la acción docente (por ejemplo, comparando información, creando o mejorando artículos, etc.), y también explica los beneficios educativos, que implican mejoras en el rendimiento académico de los estudiantes. Asimismo, se ve cómo puede constituirse como una herramienta interesante para trabajar diversas competencias asociadas a muchos estudios universitarios (por ejemplo, el trabajo colaborativo y en equipo, la escritura y comprensión de textos, análisis y síntesis, etc.).

Como la valoración general de las experiencias es mayoritariamente positiva, esta guía puede ser una herramienta muy útil en el momento de tomar la decisión de usar Wikipedia en la educación superior. Puede constituirse como una referencia importante a la hora de pensar en el diseño de nuevos cursos o a la hora de definir nuevas estrategias docentes.

\section{Referencias bibliográficas}

AIBAR, E.; LLADÓS, J.; MESEGUER, A.; MINGUILLON, J.; LERGA, M. (2015). «Wikipedia at University: what faculty think and do about it». The Electronic Library. Núm. 33(4), en prensa.

BAYLISS, G. (2013). «Exploring the cautionary attitude toward Wikipedia in higher education: implications for higher education institutions». New Review of Academic Librarianship. Núm. 19(1), págs. 36-57.

BLACK, E. W. (2008). «Wikipedia and academic peer-review: Wikipedia as a recognized medium for scholarly publication?». Online Information Review. Núm. 32(1), págs. 73-88.

CHEN, H.-L. (2010). «The perspectives of higher education faculty on Wikipedia». The Electronic Library. Núm. 28(3), págs. 361-373.

DOOLEY, P. L. (2010). «Wikipedia and the two-faced professoriate». En: Proceedings of the 16th International Symposium on Wikis and Open Collaboration (Wikisym'10). Nueva York: ACM.

EIJKMAN, H. (2010). «Academics and Wikipedia: reframing Web 2.0+ as a disruptor of traditional academic power-knowledge arrangements». Campus-Wide Information Systems. Núm. 27(3), págs. 173-185.

JASCHIK, S. (2007, 26 de enero). «A stand against Wikipedia». Inside Higher. Disponible en: http://www.insidehighered.com.

LLADÓS, J.; AIBAR, E.; LERGA, M.; MESEGUER, A.; MINGUILLÓN, J. (2013). «An Empirical Study on Faculty Perceptions and Teaching Practices of Wikipedia». En: AUGIER, M.; CIUSSI, M. Proceedings of the 12th European Conference on e-Learning. Sophia Antipolis: Academic Conferences and Publishing International Limited. Págs. 258-265.

MESEGUER-ARTOLA, A. (2014). «Learning by comparing with Wikipedia: the value to students' learning». RUSC. Universities and Knowledge Society Journal. Núm. 11(2), págs. 55-65. doi http://dx.doi.org/10.7238/rusc. v11i2.2042 
MESEGUER-ARTOLA, A.; AIBAR, E.; LLADÓS, J.; MINGUILLÓN, J.; LERGA, M. (2015). «Factors that influence the teaching use of Wikipedia in Higher Education». Journal of the Association for Information Science and Technology. (En prensa).

WANNEMACHER, K.; SCHULENBURG, F. (2010). «Wikipedia in Academic Studies: Corrupting or Improving the Quality of Teaching and Learning?». En: EBNER, M.; SCHIEFNER, M. (eds.). Looking Toward the Future of Technology-Enhanced Education: Ubiquitous Learning and the Digital Native. Hershey, PA: IGI Global. Págs. 295-310.

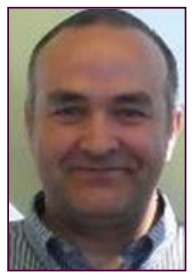

\section{Antoni Meseguer-Artola}

ameseguer@uoc.edu

\section{Profesor de los Estudios de Economía y Empresa (UOC)}

Doctor en Ciencias Económicas y Empresariales y máster en Análisis económico por la Universidad Autónoma de Barcelona (UAB). Es también licenciado en Matemáticas por la Universidad de Barcelona (UB). Ha sido profesor asistente en la UAB y actualmente es también profesor asociado en la UB. En la UOC ha sido director de la licenciatura de Ciencias del Trabajo (2001-2006), y director de los Estudios de Economía y Empresa (2006-2010). Como miembro del grupo de investigación ONE del IN3, es autor de diversos artículos y conferencias sobre competencia en precios, comercio electrónico y e-learning. Es también autor de manuales docentes de matemáticas y estadística.

Los textos publicados en esta revista están -si no se indica lo contrario- bajo una licencia Reconocimiento-Sin obras derivadas 3.0 España de Creative Commons. Puede copiarlos, distribuirlos y comunicarlos públicamente siempre que cite su autor y la revista y la institución que los publica (autoría, nombre de la revista, institución editora); no haga con ellos obras derivadas. La licencia completa se puede consultar en http://creativecommons.org/licenses/by-nd/3.0/es/deed.es.

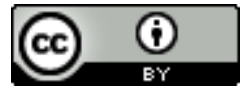

